\title{
Applications of a robotic stereotactic arm for pediatric epilepsy and neurooncology surgery
}

\author{
Brandon A. Miller, MD, PhD, Afshin Salehi, MD, David D. Limbrick Jr., MD, PhD, and \\ Matthew D. Smyth, MD
}

Department of Neurosurgery, Washington University School of Medicine in St. Louis, Missouri

\begin{abstract}
OBJECTIVE The ROSA device is a robotic stereotactic arm that uses a laser system to register the patient's head or spine with MR or CT images. In this study, the authors analyze their experience with this system in pediatric neurosurgical applications and present selected cases that exemplify the usefulness of this system.

METHODS The authors reviewed all cases that utilized the ROSA system at their institution. Patient demographics, pathology, complications, electrode placement, laser ablation, and biopsy accuracy were analyzed. Patient disposition and condition at follow-up were also analyzed.
\end{abstract}

RESULTS Seventeen patients underwent 23 procedures using the ROSA system. A total of 87 electroencephalography electrodes were placed, with $13 \%$ deviating more than $3 \mathrm{~mm}$ from target. Six patients underwent stereotactic needle biopsy, and 9 underwent laser interstitial thermotherapy (LITT). One patient who underwent LITT required a subsequent craniotomy for tumor resection. Another patient experienced an asymptomatic extraaxial hematoma that spontaneously resolved. No patient suffered neurological complications during follow-up. Follow-up from the last procedure averaged 180 days in epilepsy patients and 309 days in oncology patients.

CONCLUSIONS The precision, ease of use, and versatility of the ROSA system make it well suited for pediatric neurosurgical practice. Further work, including long-term analysis of results and cost-effectiveness, will help determine the utility of this system and if its applications can be expanded.

https://thejns.org/doi/abs/10.3171/2017.5.PEDS1782

KEY WORDS epilepsy; laser ablation; depth electrodes; stereo-EEG; robotic neurosurgery; surgical technique

$\mathrm{T}$ HE ROSA system (Zimmer Biomet/Medtech Surgical) is a computer-controlled robotic arm used for frameless stereotactic surgery. The system software allows for planning of multiple trajectories (for implantation of catheters, biopsy needles, and laser probes) using both CT scanning and MRI. The robotic arm is used to register imaging studies to patients' facial features using laser scanning. The arm is then computer driven to the appropriate location, and alignment is maintained for surgical instruments to be directed along the planned trajectory. This system can be used for precision implantation of depth electrodes,${ }^{10}$ laser interstitial thermotherapy (LITT) catheters, frameless stereotactic biopsy, ${ }^{9}$ spinal fusion, ${ }^{11}$ and endoscopic third ventriculostomy (ETV) ${ }^{7,13}$ Because more institutions are adopting this system, published literature on its applications and outcomes is growing. However, to date little has been published that illustrates the system's utility in pediatric neurosurgery. The system's ability to allow for less-invasive procedures with minimal blood loss makes it ideal for neurosurgical applications in pediatric patients.

Our institution has used the ROSA system in epilepsy surgery for stereo-electroencephalography (stereo-EEG) and laser ablation as well as neurooncology procedures, including biopsy and LITT, since February 2016. This report includes all patients who underwent ROSA-assisted 


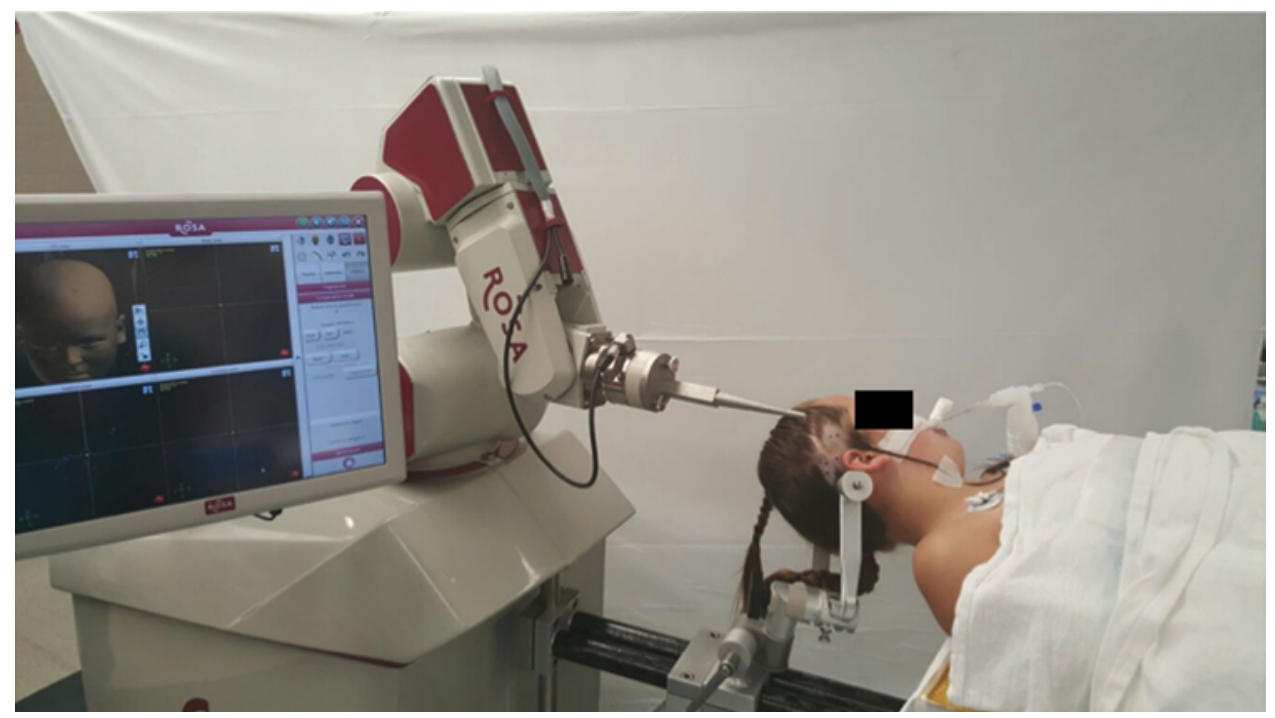

FIG. 1. Operating room configuration of the ROSA system. The patient's head is secured in a Mayfield head holder that is attached at a fixed position to the ROSA robot. The robotic arm can freely move around the patient's head to the entry point of planned stereo-EEG trajectories. In this case, all planned trajectories are in the right temporal region.

procedures through December 2016. We have achieved good outcomes with this system and have refined our practice over the course of our experience. We have found the ROSA system to be versatile and well suited to pediatric epilepsy and oncology cases in which precision electrode and catheter targeting is essential.

\section{Methods}

We retrospectively reviewed all cases in which the ROSA system was used at our institution (Washington University School of Medicine in St. Louis). All patients who had undergone 1 or more ROSA-assisted procedures were included; no patient who had undergone a ROSAassisted procedure prior to submission of the manuscript was omitted. Patient history, demographics, surgical indication, and prior surgical procedures were analyzed. We categorized patients into epilepsy and oncology categories based on the primary indication for ROSA-assisted surgery. Some patients in the seizure group did have lowgrade lesions that were surgically treated because the patient presented with a seizure disorder. Since seizure control rather than oncological diagnosis or treatment was the primary indication for surgery, these patients were analyzed in the epilepsy group.

For epilepsy cases, the number and placement of electrodes, surgical time, and subsequent clinical course were analyzed. Electrode placement accuracy was assessed by merging postoperative CT scans with preoperative scans that showed the planned trajectories. Each planned trajectory was traced in its entire axial plane, and any electrode deviating more than $3 \mathrm{~mm}$ at any point in that axial plane was categorized as inaccurate.

For oncology patients, prior operations, imaging studies, and pathological findings were analyzed. Operative time, last follow-up, and outcome were analyzed.

\section{Surgical Planning and Technique}

Each patient's treatment plan was reviewed by the senior author (M.D.S.) and the relevant multidisciplinary team. All epilepsy patients underwent multidisciplinary review prior to any operation, and oncology patients underwent multidisciplinary review postoperatively or preoperatively as deemed appropriate by their neurosurgeon.

The ROSA system allows for preoperative planning of multiple electrode or catheter trajectories on the robotic device or a stand-alone laptop workstation. All ROSA procedures are planned prior to the day of surgery. MRI and CT/CT angiography data are merged to allow for optimal delineation of pathology and intracranial vasculature. Planning times vary widely based on trajectory number, patient anatomy, and case complexity and thus were not recorded. The senior author (M.D.S.) made all biopsy plans, and depth electrode plans were made by the senior author in collaboration with an epileptologist. Each trajectory was planned to avoid vasculature and minimize transgression of eloquent structures en route to target. Extra care was taken to avoid surface cortical vessels for each trajectory in the planning phase. In the operating room, the patient was positioned in a Mayfield head holder that was fixed to the ROSA system (Fig. 1). The fixed orientation of the patient's head to the robot allows for precise stereotaxy once registration is complete. Registration was done using a semiautomated program built into the ROSA system that uses a laser at the end of the robotic arm to scan the patient's facial contours and register to the prior highdefinition 3D CT volumetric images. After registration, the patient was prepared and draped, and the robotic arm was automatically driven into alignment with each trajectory. The arm can be manually adjusted along the axis of the trajectory to achieve a comfortable working distance. The software gave the exact distance to target from the 
TABLE 1. Epilepsy patient outcomes

\begin{tabular}{ccccccccccc}
\hline $\begin{array}{c}\text { Case } \\
\text { No. }\end{array}$ & $\begin{array}{c}\text { Age at } \\
\text { Op (yrs) }\end{array}$ & $\begin{array}{c}\text { Pathological } \\
\text { Dx }\end{array}$ & $\begin{array}{c}\text { Stereo-EEG } \\
\text { Op Time (mins) }\end{array}$ & $\begin{array}{c}\text { No. of } \\
\text { Electrodes }\end{array}$ & $\begin{array}{c}\text { No. of Missed } \\
\text { Electrodes }\end{array}$ & $\begin{array}{c}\text { Stereo-EEG } \\
\text { Duration (days) }\end{array}$ & $\begin{array}{c}\text { Days to } \\
\text { Op }\end{array}$ & $\begin{array}{c}\text { Follow-Up } \\
\text { Treatment }\end{array}$ & $\begin{array}{c}\text { Engel } \\
\text { Duration (days) } \\
\text { Class }\end{array}$ \\
\hline 1 & 6 & TSC & 98 & 10 (bilat) & 1 & 2 & 56 & Craniotomy & 113 & IV \\
\hline 2 & 12 & LGG & 50 & 6 & 2 & 3 & 29 & LITT & 146 & III \\
\hline 3 & 6 & FCD & 56 & 8 & 0 & 2 & 58 & Craniotomy & 90 & I \\
\hline 4 & 18 & FCD & 97 & 10 & 1 & 3 & 133 & Neuropace & 104 & III \\
\hline 5 & 20 & FCD & 77 & 7 & 1 & 7 & 72 & LITT & 326 & I \\
\hline 6 & 15 & FCD & 130 & 9 & 2 & 7 & 57 & LITT & 212 & I \\
\hline 7 & 10 & FCD & 90 & 7 & 0 & 3 & 51 & LITT+biopsy & 329 & II \\
\hline 8 & 6 & TSC & 71 & 8 & 2 & 5 & 23 & Craniotomy & 237 & IV \\
\hline 9 & 12 & None & 214 & 12 & 0 & 5 & Pending* & Gridt & NA & NA \\
\hline $10 \ddagger$ & 6 & NA & NA & NA & NA & NA & NA & LITT & ITT & II \\
\hline 11 & 15 & TSC & 94 & 10 (bilat) & 0 & 7 & 72 & Craniotomy & 59 & I \\
\hline
\end{tabular}

Dx = diagnosis; FCD = focal cortical dysplasia; $L G G$ = low-grade glioma; NA = not applicable; TSC = tuberous sclerosis complex.

* This patient has not yet returned for treatment surgery.

t Planned surgery for additional seizure localization.

$\ddagger$ This patient underwent LITT therapy for completion of a prior corpus callosotomy.

robot arm, allowing for calculation of the correct depth to pass electrodes or catheters. Typically, we drove the robotic arm to the desired trajectory and used it to stabilize a battery-powered twist drill with which we simultaneously puncture the skin and make a 2.4-mm pilot hole for an anchor bolt. We then aligned the robotic arm to the very back of the anchor bolt and recorded the distance from the end of the bolt to the target. This gave us the depth to which electrodes/catheters were to be passed into the anchor bolt to their target. Prior to inserting depth electrodes, we passed a semirigid stylet to the desired depth to make a track for the electrode. After electrode placement, patients underwent $\mathrm{CT}$ scanning prior to extubation and room turnover. Biopsy patients were extubated in the operating room once an adequate pathological specimen is verified.

Laser ablation with the ROSA system has been described elsewhere. ${ }^{5}$ Our laser ablation technique utilizes the same principles as our preparation for stereo-EEG implantation. We perform all laser ablations in an intraoperative MRI suite, which allows for LITT probe placement and laser ablation in the same location. Our institution's laser ablation technique has been described elsewhere. ${ }^{1}$

\section{Results \\ Epilepsy Patients}

Eleven patients underwent ROSA-assisted procedures for epilepsy (Table 1). The average patient age was 11.6 years. Ten patients underwent depth electrode implantation for stereo-EEG recording, and 1 (Case 10) underwent laser ablation of the posterior third of the corpus callosum after she had undergone an anterior corpus callosotomy years earlier. Nine of the patients who underwent stereo-EEG had lesional pathology that guided electrode placement (Table 1). All epilepsy patients had undergone noninvasive monitoring at some point in their preoperative workup. An average of 8.7 electrodes were placed per patient (range 6-12). Two patients with tuberous sclerosis complex (Cases 1 and 11) underwent bilateral electrode placement. The surgical duration averaged 98 minutes (range 50-214 minutes). The average time per electrode placed was 10.9 minutes, calculated as total surgical time divided by the number of electrodes. The actual time to place each electrode, from incision to final anchor bolt tightening, was approximately 5-7 minutes, based on the timing of a limited number of electrode placements during our early experience. A total of 87 electrodes were placed, with $9(10 \%)$ electrodes (mean 1.1 per case, range $0-2$ per case) being more than $3 \mathrm{~mm}$ off target at any point along the planned trajectory. Of those electrodes that deviated more than $3 \mathrm{~mm}$ from the planned trajectory, the average greatest distance form intended trajectory was 5 $\mathrm{mm}$ (range 3.9-8.5 $\mathrm{mm}$ ). The average duration of invasive monitoring was 4.4 days (range $2-7$ days). One patient (Case 11) developed an asymptomatic extraaxial hematoma as a result of electrode placement. This was detected on the immediate postoperative CT scan. The patient underwent observation in the ICU for 1 night, and repeat CT scanning showed resolution of the hematoma.

Ten patients have thus far undergone surgery for epilepsy treatment. Nine had previously undergone stereo-EEG placement, and 1 underwent LITT for corpus callosotomy completion without prior stereo-EEG. Other epilepsy treatment procedures included open resection, ROSAguided responsive neurostimulator (Neuropace) placement, laser ablation, or biopsy and laser ablation of epileptic focus (Table 1). The patient who underwent responsive neurostimulator implantation had electrodes placed in the inferior frontal lobe, in the hand and face primary motor areas. Because of the eloquent nature of her seizure focus, this patient was a poor candidate for resective surgery. There were no complications from any treatment procedure. The time between electrode removal and treatment surgery ranged between 23 and 133 days, allowing time 
TABLE 2. Oncology patient outcomes

\begin{tabular}{cclclll}
\hline Case No. & Age at Op (yrs) & \multicolumn{1}{c}{ Procedure } & Op Time (mins) & Pathology & Outcome & Follow-Up Duration (days) \\
\hline 12 & 5 & Biopsy & 60 & LGG & Chemotherapy & 157 \\
\hline $13^{*}$ & 10 & Biopsy followed by LITT & 33 & Immature teratoma & Required craniotomy & 385 \\
\hline 14 & 17 & Biopsy/LITT & 105 & Oligodendroglioma & No recurrence & 393 \\
\hline 15 & 14 & Biopsy/LITT & 126 & LGG & No recurrence & 387 \\
\hline 16 & 14 & Biopsy/LITT & 206 & SEGA & No recurrence & 340 \\
\hline 17 & 15 & LITT & 199 & JPA & No recurrence & 196 \\
\hline
\end{tabular}

JPA = juvenile pilocytic astrocytoma; SEGA = subependymal giant cell astrocytoma .

* Patient required craniotomy for tumor resection after laser ablation.

for elective review of the stereo-EEG data with the epilepsy team and elective scheduling of the treatment procedure at a mutually agreeable time for the surgical team and the family. One patient will require further invasive monitoring with grid electrodes before treatment. Of the 10 patients who underwent surgery, 6 have achieved Engel Class I or II outcomes (Table 1). ${ }^{3}$

\section{Oncology Patients}

Six patients (average age 12.5 years) underwent ROSAassisted biopsy and/or LITT for diagnosis or treatment of oncological disease (Table 2). The average operative time
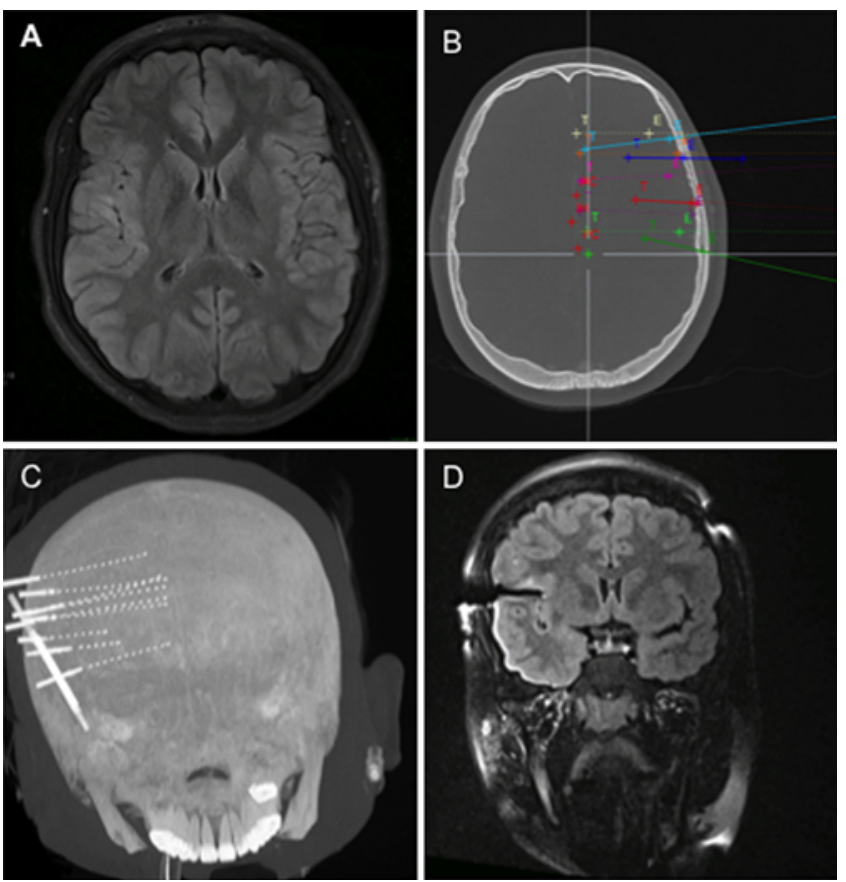

FIG. 2. Case 6. A: Axial FLAIR sequence MR image demonstrating subtle hyperintensity in the right pars opercularis region. B: Axial image from ROSA software demonstrating planned trajectories (left and right are opposite of convention on this projection). C: Coronal CT 3D maximum intensity projection scan obtained after ROSA-guided stereo-EEG electrode placement. D: Coronal intraoperative MR image obtained before LITT, demonstrating accurate targeting of the region of interest. was 122 minutes. All patients who underwent biopsy and LITT underwent both procedures in the same setting using 1 trajectory. One patient who underwent LITT without biopsy had diagnosis of juvenile pilocytic astrocytoma from a prior surgery (Case 17). There were no complications for any procedure. Of the 5 patients who underwent LITT, 1 required subsequent craniotomy for lesion resection (Case 13, see Illustrative Cases). No others have experienced tumor progression to date.

\section{Illustrative Cases}

Case 6: Stereo-EEG and Laser Ablation for Epilepsy

This 15 -year-old boy had medically refractory epilepsy with an onset in early childhood (Fig. 2). The patient had an average of 1 seizure per day despite medical therapy. Noninvasive EEG recordings suggested a right frontal seizure onset. He had an area of FLAIR signal in the right frontal lobe including the pars opercularis, consistent with cortical dysplasia. He underwent implantation of 9 depth electrodes in the right frontal lobe. After 7 days, the electrodes were removed after capturing seizure onset at the area of signal abnormality. During invasive monitoring, the electrode in the seizure focus was stimulated without speech or motor arrest.

Approximately 2 months after the invasive mapping hospitalization, the patient underwent stereotactic laser ablation of the epileptic focus using the ROSA system. There were no complications during any procedure. The patient's recoveries after both hospitalizations were uneventful. Seven months after laser ablation he was seizure free, with improved behavior and alertness.

\section{Case 13: Biopsy and Laser Ablation for Tumor}

This 10-year-old boy presented with hydrocephalus and altered mental status (Fig. 3). Initial MRI showed aqueductal stenosis but no mass lesion. The patient underwent successful ETV and was discharged from the hospital after making an uneventful recovery.

Approximately 15 months after ETV, the patient underwent surveillance MRI, which revealed a pineal region mass. He then underwent ROSA-guided biopsy followed by laser ablation of the lesion with the ROSA system while in the intraoperative MRI scanner. The biopsy result was mature teratoma. The patient made an uneventful recovery and was discharged home from the hospital. Surveil- 

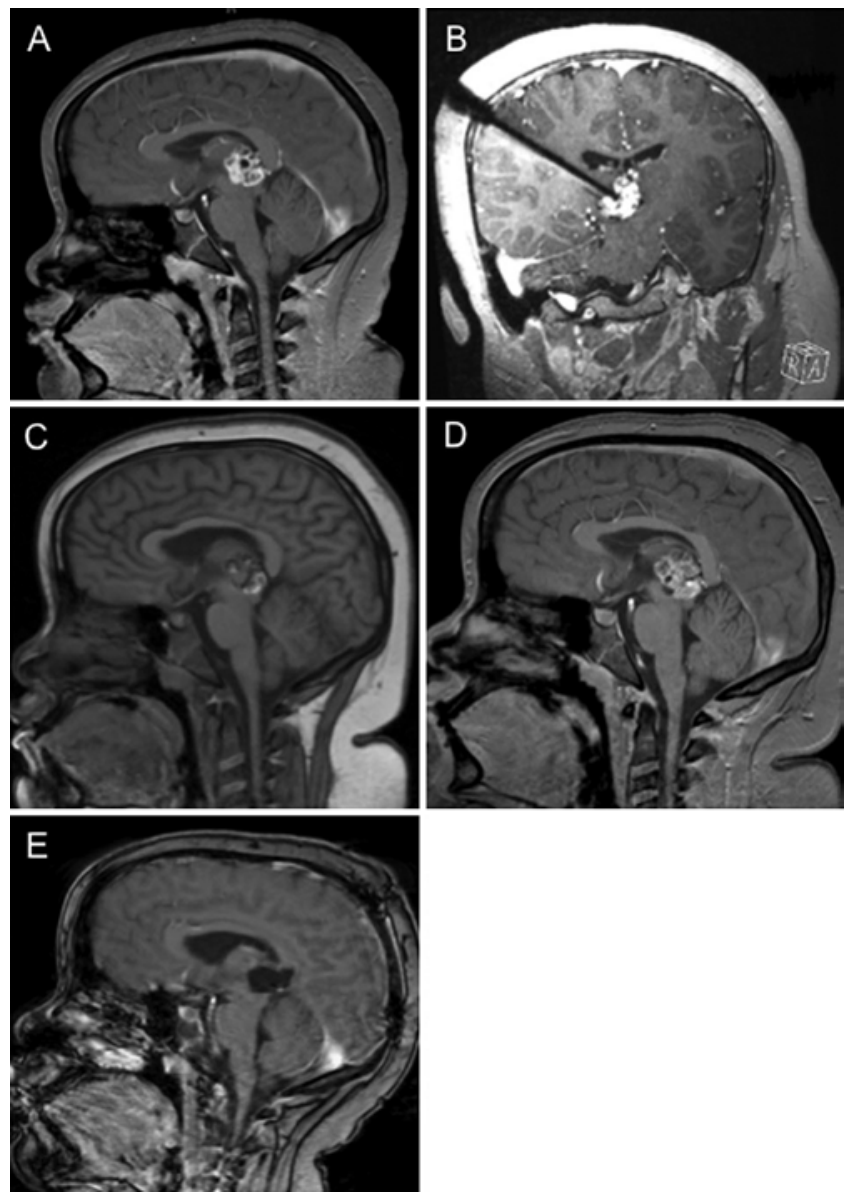

FIG. 3. Case 13. A: Sagittal T1-weighted contrast-enhanced MR image obtained before LITT, revealing an enhancing lesion in the pineal region. B: Coronal intraoperative MR image obtained after ROSA-guided laser probe placement confirming accurate targeting. C: Sagittal MR image obtained 1 month after LITT, demonstrating partial treatment of the teratoma. D: Sagittal MR image obtained 5 months after LITT, showing tumor recurrence. E: Sagittal MR image obtained 2 months postcraniotomy for tumor gross-total resection.

lance imaging performed 5 months after laser ablation revealed lesion growth; therefore, the decision was made to proceed with open resection. The patient underwent interhemispheric transcallosal resection of the mass, and gross-total resection was achieved. Final pathological examination findings indicated an immature teratoma. The patient made an uneventful recovery from surgery and underwent conformal external-beam radiation therapy.

\section{Case 2: Stereo-EEG and Laser Ablation After Tumor Resection}

This 12-year-old girl had undergone partial resection of a low-grade glioma in the frontotemporal lobe that presented with seizures 2 years earlier (Fig. 4). The lesion extended into the insula, inferior frontal lobe, and basal ganglia. The patient initially achieved seizure freedom after resection but then developed recurrent seizures. Six stereo-EEG electrodes were placed, 4 around the lesion margin and 2 in the insula. Ictal and interictal EEG recordings showed the seizure focus to be within the insula
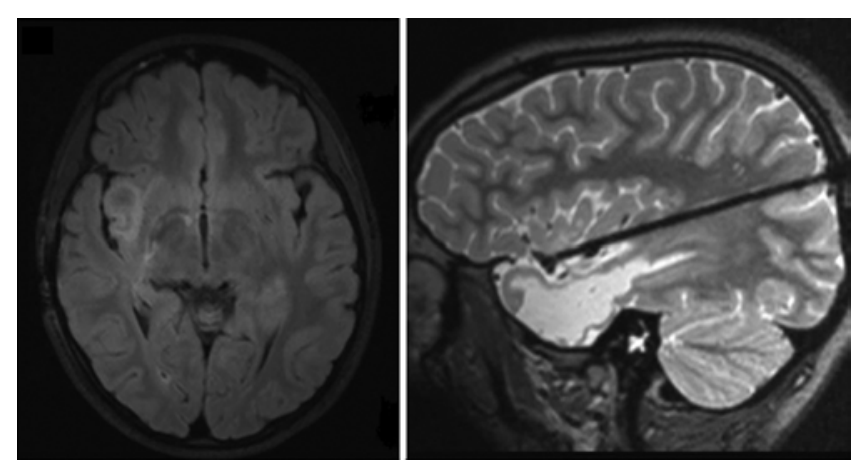

FIG. 4. Case 2. Left: Axial FLAIR MR image demonstrating residual insular tumor. Stereo-EEG determined this to be the region of seizure onset. Right: Intraoperative sagittal image obtained before LITT, demonstrating the accurate targeting of the region of interest.

and not from the prior temporal resection bed. The patient underwent laser ablation of the insula with no complications and initial seizure improvement. Long-term followup is pending.

\section{Discussion}

Using the ROSA system for stereotactic guidance, we placed 87 total EEG electrodes; no symptomatic complications were associated with electrode placement. Eleven electrodes were off target by more than $3 \mathrm{~mm}$ at any point in the trajectory; however, there was no need for implantation of additional electrodes to successfully localize seizure foci. This is lower than a roughly $13 \%$ complication rate associated with subdural grid and strip electrode placement at our institution. ${ }^{8}$ We noted 2 electrodes that took a significant bend in trajectory despite a well-positioned anchor bolt. Both occurred in patients with tuberous sclerosis (Cases 1 and 8), and we suspect that a deflection occurred due to the density change of the tissue at the tuber/normal brain interface. Our cutoff for electrode inaccuracy ( $3 \mathrm{~mm}$ from the target) was chosen based on our own preferences and not based on data showing a critical cutoff for electrode accuracy necessary to achieve localization and good outcomes. While more precise electrode placement is always preferable, our data demonstrate that small deviations from the original stereo-EEG plan do not necessarily lead to poor outcomes. Prior to ROSA stereo-EEG, we had previously placed stereo-EEG electrodes during craniotomies for grid placement using either optical or electromagnetic stereotactic guidance. The small number of electrodes placed with our prior technique could not be meaningfully compared with the use of stereo-EEG described in this paper. For LITT, prior to adopting ROSA, we used a number of strategies for thermal probe guidance, which we have discussed previously. ${ }^{2}$

A recent series utilizing the Leksell stereotactic frame for stereo-EEG electrode placement in 122 patients (1586 electrodes total) reported a $0.18 \%$ chance of complication per electrode and morbidity of $2.5 \%$ (3 of 122 patients). ${ }^{4}$ In another study examining different electrode types, complications associated with depth electrodes occurred in 1 of 14 patients. ${ }^{6}$ One patient in our study experienced 
an asymptomatic extraaxial hemorrhage after depth electrode placement. This corresponds to a hemorrhage rate of $9 \%$ for all stereo-EEG procedures. Assuming that the hemorrhage was due to a single electrode pass, this would constitute a rate of 1 hemorrhage per 87 electrode passes, or $1.1 \%$. With no complications from symptomatic hemorrhage, infection, or need for electrode replacement, along with an acceptable duration of invasive monitoring (mean 4.1 days), ROSA-assisted stereo-EEG placement compares favorably to open grid placement and other forms of stereo-EEG implantation. Others have shown the ROSA system to be safe for stereotactic biopsy. A recent summary of 100 biopsies reported only 2 cases of transient deterioration related to hemorrhage. ${ }^{9}$ In our experience, none of the 6 patients who underwent biopsies, 4 of which were followed by LITT, experienced any hemorrhage or other complications.

As with any new technology, enthusiasm must be tempered by an understanding of the device's limitations. Therefore, we have designed and modified our practice to overcome to adapt the nuances of the ROSA system and simplify our workflow. The mean surgery duration for our 11 stereo-EEG patients was 98 minutes. This is comparable to 107 minutes per stereo-EEG case in another group's experience. ${ }^{4}$ We have noticed an increase in operative efficiency as our team becomes more familiar with the ROSA workflow. We find our stereo-EEG strategy to be effective for guiding resective and ablative treatments for epilepsy patients, as 10 of 11 patients have gone on to treatment surgery. This compares favorably with another group's recent experience with $77 \%$ of stereo-EEG patients going on to resective surgery. ${ }^{12}$

We attribute our low complication rate and low rate of electrode inaccuracy to several factors. Planning for each trajectory was done in detail several days prior to surgery for all cases. In several instances vascular anatomy was encountered, requiring multiple modifications of trajectories, and trial and error was needed to determine a safe trajectory. Maintaining an orthogonal trajectory to the skull minimized skiving of the drill or failure of the anchor bolt to thread properly. There is a small amount of movement allowed when the drill is within the drill guide, so care must be taken to avoid user error and slightly altering the trajectory.

To prevent postoperative complications, petroleum jelly gauze strips are packed around the anchor bolt on the back of the patient's head to build a base to prevent focal pressure against the bolt when the patient lies in bed. Using our methods we have not experienced any bolt pullout or loss of purchase in the skull. A specific preoperative CT protocol is used for obtaining the registration images in which the entire face, free of any foreign objects or compression of facial features, is captured. Furthermore, a postoperative thin-cut CT scan is obtained immediately following the surgery. This is used for both electrode placement error calculation and assessment for any intracranial hemorrhage. A decision for postoperative observation in the ICU setting versus floor is made after review of the CT scans. Initially, CT scanning was performed prior to extubation, but, given that there were no symptomatic hemorrhages, we now perform the extubation prior to CT scanning. Fi- nally, electrode removal is typically done under general anesthesia with a laryngeal mask airway. All patients were discharged on the day of removal or the following day.

\section{Conclusions}

Our experience with the ROSA system for stereo-EEG, biopsy and LITT demonstrates it to be a useful adjunct for pediatric neurosurgical practice. While results are encouraging, our study is limited by short follow-up and its retrospective nature. We are continuing to analyze our results as more procedures and longer follow-up are completed.

\section{Acknowledgments}

We thank Dr. Monica Chau for her assistance with figures and critical reading of the manuscript.

\section{References}

1. Dadey DY, Kamath AA, Leuthardt EC, Smyth MD: Laser interstitial thermal therapy for subependymal giant cell astrocytoma: technical case report. Neurosurg Focus 41(4):E9, 2016

2. Dadey DY, Kamath AA, Smyth MD, Chicoine MR, Leuthardt EC, Kim AH: Utilizing personalized stereotactic frames for laser interstitial thermal ablation of posterior fossa and mesiotemporal brain lesions: a single-institution series. Neurosurg Focus 41(4):E4, 2016

3. Engel J Jr, Van Ness PC, Rasmussen TB, Ojemann LM: Outcome with respect to epileptic seizures, in Engel J Jr (ed): Surgical Treatment of the Epilepsies, ed 2. New York: Raven Press, 1993, pp 609-621

4. Gonzalez-Martinez J, Mullin J, Vadera S, Bulacio J, Hughes $\mathrm{G}$, Jones S, et al: Stereotactic placement of depth electrodes in medically intractable epilepsy. J Neurosurg 120:639-644, 2014

5. Gonzalez-Martinez J, Vadera S, Mullin J, Enatsu R, Alexopoulos AV, Patwardhan R, et al: Robot-assisted stereotactic laser ablation in medically intractable epilepsy: operative technique. Neurosurgery 10 (Suppl 2):167-173, 2014

6. Hedegärd E, Bjellvi J, Edelvik A, Rydenhag B, Flink R, Malmgren K: Complications to invasive epilepsy surgery workup with subdural and depth electrodes: a prospective population-based observational study. J Neurol Neurosurg Psychiatry 85:716-720, 2014

7. Hoshide R, Calayag M, Meltzer HS, Levy ML, Gonda DD: Robot-assisted endoscopic third ventriculostomy. Neurosurgery 63 (Suppl 1):204, 2016 (Abstract)

8. Johnston JM Jr, Mangano FT, Ojemann JG, Park TS, Trevathan E, Smyth MD: Complications of invasive subdural electrode monitoring at St. Louis Children's Hospital, 19942005. J Neurosurg 105 (5 Suppl):343-347, 2006

9. Lefranc M, Capel C, Pruvot-Occean AS, Fichten A, Desenclos C, Toussaint P, et al: Frameless robotic stereotactic biopsies: a consecutive series of 100 cases. J Neurosurg 122:342-352, 2015

10. Mullin JP, Smithason S, Gonzalez-Martinez J: Stereoelectro-encephalo-graphy () with robotic assistance in the presurgical evaluation of medical refractory epilepsy: a technical note. J Vis Exp (112):e53206 2016

11. Schatlo B, Molliqaj G, Cuvinciuc V, Kotowski M, Schaller K, Tessitore E: Safety and accuracy of robot-assisted versus fluoroscopy-guided pedicle screw insertion for degenerative diseases of the lumbar spine: a matched cohort comparison. J Neurosurg Spine 20:636-643, 2014

12. Serletis D, Bulacio J, Bingaman W, Najm I, González- 
Martínez J: The stereotactic approach for mapping epileptic networks: a prospective study of 200 patients. J Neurosurg 121:1239-1246, 2014

13. Zimmermann M, Krishnan R, Raabe A, Seifert V:

Robot-assisted navigated endoscopic ventriculostomy: implementation of a new technology and first clinical results. Acta Neurochir (Wien) 146:697-704, 2004

\section{Disclosures}

Dr. Limbrick states that he receives non-study-related clinical/ research support from Medtronic, Karl Storz Inc., and Microbot Medical Inc.

\section{Author Contributions}

Conception and design: Smyth, Miller. Acquisition of data: all authors. Analysis and interpretation of data: all authors. Drafting the article: Miller, Salehi. Critically revising the article: all authors. Reviewed submitted version of manuscript: all authors. Statistical analysis: Miller. Administrative/technical/material support: Smyth, Limbrick. Study supervision: Smyth, Limbrick.

\section{Supplemental Information}

\section{Previous Presentations}

An interim analysis of these data was presented at the 45th Annual Meeting of the AANS/CNS Section on Pediatric Neurological Surgery, December 5-8, 2016, Orlando, Florida.

\section{Correspondence}

Matthew D. Smyth, Department of Neurosurgery, Washington University School of Medicine, One Children's Pl., Ste. 4s20, CB 8057, St. Louis, MO 63110. email: smythm@wudosis.wustl.edu. 